\title{
Sobre el estado de la cuestión de las políticas públicas en salud mental en América Latina: Una revisión sistemática
}

\section{Resumen:}

Este trabajo propone realizar una revisión sistemática sobre el estado de la cuestión de las políticas públicas en el campo de la salud mental en América Latina, para describir los abordajes teóricos y metodológicos. La revisión sistemática, según la Declaración PRISMA, selleva a cabo en Agosto de 2020 y se seleccionan veinticinco artículos científicos provenientes de 6 bases de datos que refieren a publicaciones en castellano entre 2010-2020, en donde se concluye la importancia de las políticas públicas en salud mental sobre temas referidos a salud pública, discapacidad, niñez e inclusión social, garantizando así los derechos humanos esenciales de cada sujeto.

Palabras clave: Salud Mental - Política Pública-Subjetividad-Revisión Sitemática

\section{Summary:}

This work proposes to carry out a systematic review on the state of the art of public policies in the field of mental health in Latin America, to describe the theoretical and methodological approaches. The systematic review, according to the PRISMA Declaration, is carried out in August 2020 and twenty-five scientific articles are selected from 6 databases that refer to publications in Spanish between 2010-2020, where the importance of public policies is concluded in mental health on issues related to public health, disability, childhood and social inclusion, thus guaranteeing the essential human rights of each subject.

Keywords: Mental Health - Public Policy - Subjectivity - Systematic Review

Nicolás Campodónico: nicolas campodonico@hotmail.com https://orcid.org/00017182-6983 CONICET / UNLP Doctor en Psicología, Especialista en Clínica Psicoanalítica con Adultos (UNLP). Docente e investigador de la cátedra de Psicopatología I de la UNLP. Profesor adjunto de la Maestría de Psicoanálisis de la Universidad Kennedy. Becario posdoctoral del CONICET. Autor de libros, capítulos de libros, artículos en revista nacionales e internacionales. Recibido 30/09/2020, Publicado 27/10/2020. 
Delimitación del objeto de estudio

El objeto de estudio del presente trabajo de investigación, de tipo de revisión sistemática, tiene como eje principal explorar el estado de la cuestión de las políticas públicas en el campo de la salud mental, en los ámbitos académicos así como las preocupaciones y recomendaciones de los expertos del tema, centrándose como zona geográfica a diferentes países de América Latina,

Este trabajo resulta una continuación directa de la investigación doctora y posdoctoral, financiada por la Universidad Nacional de La Plata y por el CONICET. En este sentido, a parti de lo trabajado en estas investigaciones previas, se podría denotar la existencia de un importante incremento de la demanda de atención psicoterapéutica en el ámbito público, tanto a nivel de los centros de salud municipales como en los servicios de Psicopatología y Salud Mental de las instituciones hospitalarias (Campodónico, 2013; 2015). Este incremento se estima en un $77 \%$ de la población con padecimiento de un trastorno mental en algún momento de su vida, por el cual consultaría a un profesional "psi", de acuerdo al Estudio Argentino de Epidemiología en Salud Mental (2017), publicado en la revista científica Social Psychiatry and Psychiatric Epidemiology, en donde busca estimar las tasas de prevalencia y severidad de los trastornos mentales a lo largo de la vida y la edad de inicio de los mismos, as como la demora en buscar asistencia y la eficiencia de los servicios. Esto coincide con la ya conocida extensión de la ofert psicoterapéutica a nivel social, extensión que condiciona a su vez la apertura de un conjunto de demandas de asistencia, al como lo señala desde el campo de la sociología, Ehrenberg (2010).

En la investigación doctoral y posdoctoral previamente mencionada, se planteó como área de vacancia una historización que pueda dar cuenta del surgimiento y configuración de las políticas públicas en salud mental en la Argentina.

A partir del trabajo realizado se ha podido abordar lo sucedido en la articulación de las políticas públicas en las décadas del '50 y '60 en la Argentin tras la profesionalización de la Psicología (Campodónico, 2018a, 2018b).

Este estudio se enmarca dentro de las áreas de la Psicología y la Salud Pública y sobre estas líneas se basará la elaboración teórica - conceptual, para dar respuesta al objetivo propuesto. Ahora bien, en el presente trabajo se plantea al 2010 como año de comienzo, que corresponde a la formulación de la Ley Nacional de Salud Mental en la Argentina, para explorar las consecuencias de la misma como también otras normativas y políticas públicas en salud mental en los países de América Latina, referido a temas tale como por ejemplo, adopción en casos de padres del mismo sexo; leyes de violencia de género, cuidados en la infancia, los protocolos de acción y cuidados ante emergencia psico-social como lo es pandemia por el COVID-19, entre otros.

Se resalta el reconocimiento del campo de la salud mental como tópico de creciente preocupación tanto a nivel de lo que es política pública como a nivel de las diferentes orientaciones teóricas. Se subraya la importancia de la salud mental en la subjetividad de cada uno de los habitantes de cada país de Latinoaméri- ca, y es por ello, que se apunta a explorar sobre el estado de la cuestión de las políticas públicas en salud mental para, de esta manera, vislumbrar los focos de atención, recomendaciones y preocupaciones d expertos del tema en el ámbito académico y en la salud pública. Se parte de suponer que existe un punto en donde la relación entre la planificación y la gestión de políticas públicas y el aporte desde la Psicología, resulta de suma importancia para ser interrogado y generar respuestas a las problemáticas emergentes en el campo de la salud pública. Este tema de investigación resulta relevante para evaluar, planificar y gestionar temas de políticas públicas de acuerdo a las problemáticas actuales de nuestra sociedad. De este modo se parte de entender el impacto, la configuración y construcción de las políticas de salud como productos históricos, asociando las transformaciones económicas y sociales con los cambios en las concepciones de los procesos de salu$\mathrm{d}$-enfermedad, los diferentes sistemas de intervención y los actores que se consideraron aptos para llevarlas a cabo. De esta manera, también se parte de comprender las diferentes lógicas que sustentan el desarrollo del sistema público de salud en general y salud mental en particular en los últimos años.

\section{Objetivo}

A través del presente estudio, se preende explorar el estado de la cuestión de las políticas públicas en salud mental en América Latina.

\section{Método}

El presente estudio se valió del uso del método PRISMA, siguiendo los pasos de calidad para la revisión sistemática. Se han utilizado 19 ítems de un total de 27 , propuestos por esta metodología.
Los ítems que no se tomaron en cuenta, fueron excluidos dado que refieren a estudios de corte metaanalítico, y difieren de los objetivos de este estudio.

Se realizó una búsqueda exhaustiva de artículos científicos publicados acerca de la implementación de las políticas públicas de la salud mental en los países de latinoamérica.

\section{Procedimiento}

Dados el objetivo de este estudio y en atención al método seleccionado, los ítems que se decidieron incluir fueron: 1 (titulo), 2 (resumen estructurado), 3 (justificación), 4 (objetivo), 6 (criterios de elegibilidad), 7 (fuentes de información), 8 (búsqueda), 9 (selección de los estudios), 10 (proceso de extracción de datos), 11 (lista de datos), 16 (análisis adicionales), 17 (selección de estudios), 18 (características de los estudios), 20 (resultados de los estudios individuales), 21 (síntesis de los resultados), 23 (análisis adicionales), 24 (resumen de la evidencia), 25 (limitaciones ) y 26 (conclusiones). Los ítems que se decidió excluir fueron: 5 (protocolo y registro), 12 (riesgo de sesgo en los estudios individuales) 13 (medidas de resumen), 14 (síntesis de resultados), 15 y 22 (riesgo de sesgo entre los estudios), 19 (riesgo de sesgo en los estudios) y 27 (financiación)

Se tuvieron en cuenta diferentes bases de datos, entre ellas: Red Iberoamericana de Innovación y Conocimiento Científico (REDIB), Scientific Electronic Library Online (SCIELO), Dialnet, Red de Revistas Científicas de América Latina y el Caribe, España y Portugal (REDALYC) y Portal Regional en Salud Organización Panamericana de la Salud, en las cuales se investigó el tratamiento que se le daba al eje de la investigación. 
En un primer momento, la temática fue abordada de forma general sin priorizar filtros específicos de interés para este trabajo. Una vez evaluada la información recolectada en las diferentes bases, se tomó la decisión de abordar la investigación exclusivamente de la mano de los filtros de búsqueda aplicados, pues los resultados obtenidos eran más rigurosos y específicos para el tema a profundizar.

\section{Términos de búsqueda}

Una vez seleccionadas las bases de datos definitivas, se hizo una primera búsqueda sobre el constructo seleccionado para realizar la búsqueda del marco de investigación, a saber: Políticas públicas en salud mental.

El operador conceptual "políticas públicas en salud mental” arrojó un tota de 4413 muestras de análisis sumando individualmente los resultados de las seis bases de datos mencionadas anteriormente.

Una vez realizada la búsqueda del tema en las bases elegidas, se comenzó a utilizar filtros específicos y aparecieron los criterios de inclusión y exclusión.

Del corpus de trabajos, la delimitación realizada incluyó: Idioma: español; Fecha de publicación: 2010-2021 Áreas de estudio: Psicología, Psicología Clínica, Ciencias de la salud, Política y servicios de salud, Políticas Públicas, Ciencias Sociales, Salud, Salud Pública, Salud Mental; Países de publicación: Argentina, Colombia, México, Cuba, Perú, Costa Rica, Chile, Venezuela, Uruguay. Los textos que se utilizarán serían artículos científicos y textos completos. Rápidamente se modificaron las cantidade luego de la aplicación de estos filtros, arrojando números considerablemen-
Diagrama de flujo te menores. Sin embargo, cabe aclarar que en cada base de datos se aplicaron los filtros propios que cada base de dato permite. En 5 de las 6 bases de datos se utilizó como concepto de búsqueda "políticas públicas en salud mental”, sobre el cual se aplicaron los filtros de cada base de dato, pero sin embargo en Dialnet se comenzó con el mismo concepto de búsqueda, pero para reducir el número se aplicó una modificación en el término de búsqueda de la siguiente manera: "políticas públicas en salud mental+salud pública+psicología”.

Una vez analizados los artículos en profundidad, se prosiguió con el cribado definitivo, teniendo en cuenta criterio de pertinencia específica a la investigación. Posteriormente se volcaron los resultados definitivos.

Respecto a las palabras claves, los porcentajes de distribución según las bases de datos han sido: Redib $(0,18 \%)$, SciELO $(0.13 \%)$, Dialnet $(0,18 \%)$ Doaj $(0,15)$, Redalyc $(0,11)$, Portal Regional en Salud Organización Panamericana de la Salud $(0,09)$.

En total y, desde un principio, se obtuvieron 4413 muestras de análisis, fueron excluidas 4375 y el total de artículos relevados fueron 38 (0,86\% del número inicial). De este número, hay 13 artículos duplicados $(0,29 \%)$ en las diferente bases de datos utilizadas. Por lo tanto se han trabajado con 25 artículos científi$\cos (0,56 \%)$.

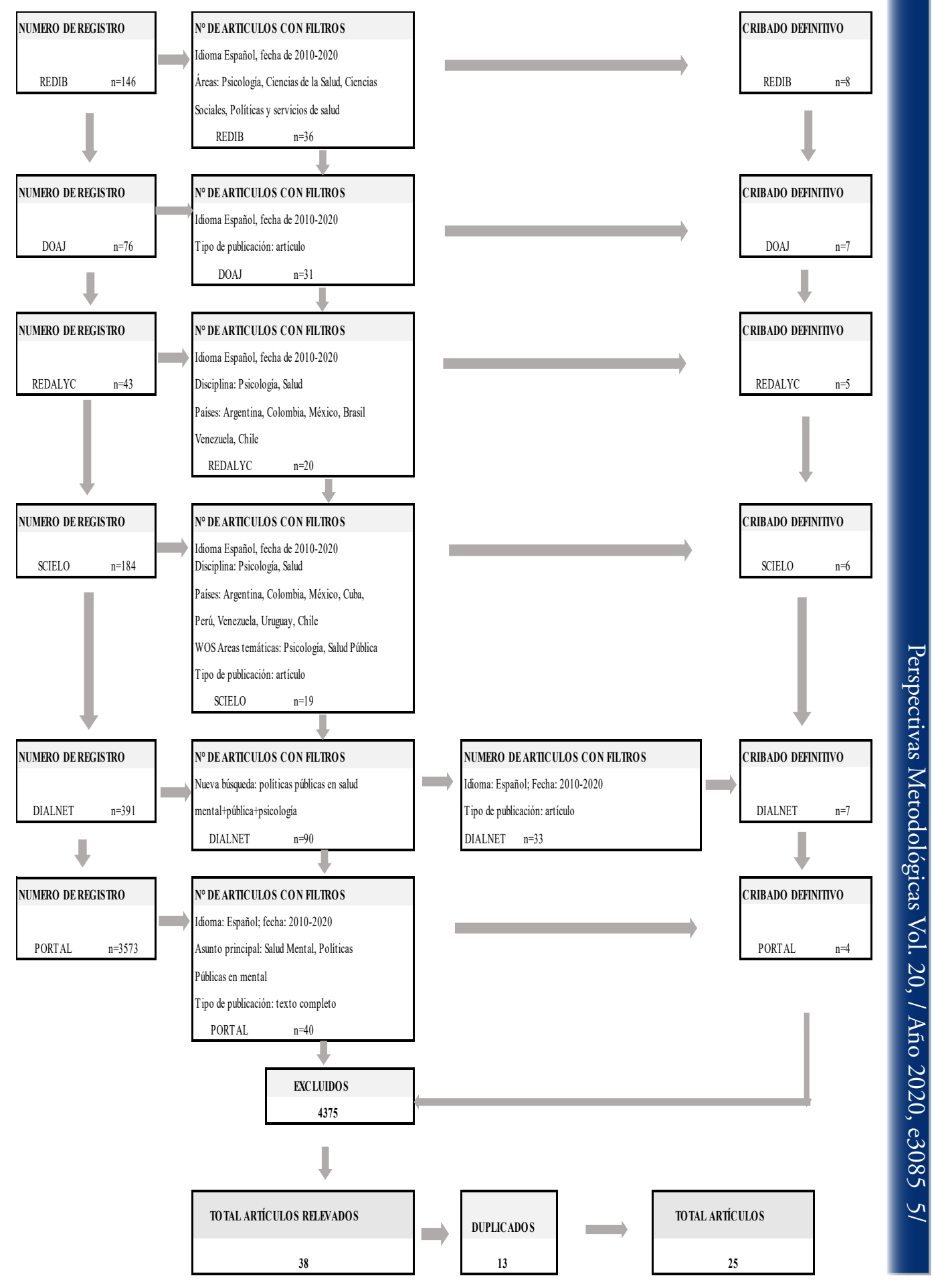

Revista Perspectivas Metodológicas | Universidad Nacional de Lanús | ISSN 2618-4125 
Tipo de

Muestra

Resultados

1 Cea Madrid "Locos por

REDIB Estudio descriptivo

comunitario. De

neo.

carácter cualitativo

participativo, se

describe una mirada

global sobre el

reconocimiento,

ejercicio y defensa de

derechos en salud

mental desde la

perspectiva de

usuarios(as) y

exusuarios(as). Se

concluye que el

protagonismo de estos

actores sociales

constituye un aspecto

central para la

distribución del poder

y la recuperación de

derechos de

ciudadanía.

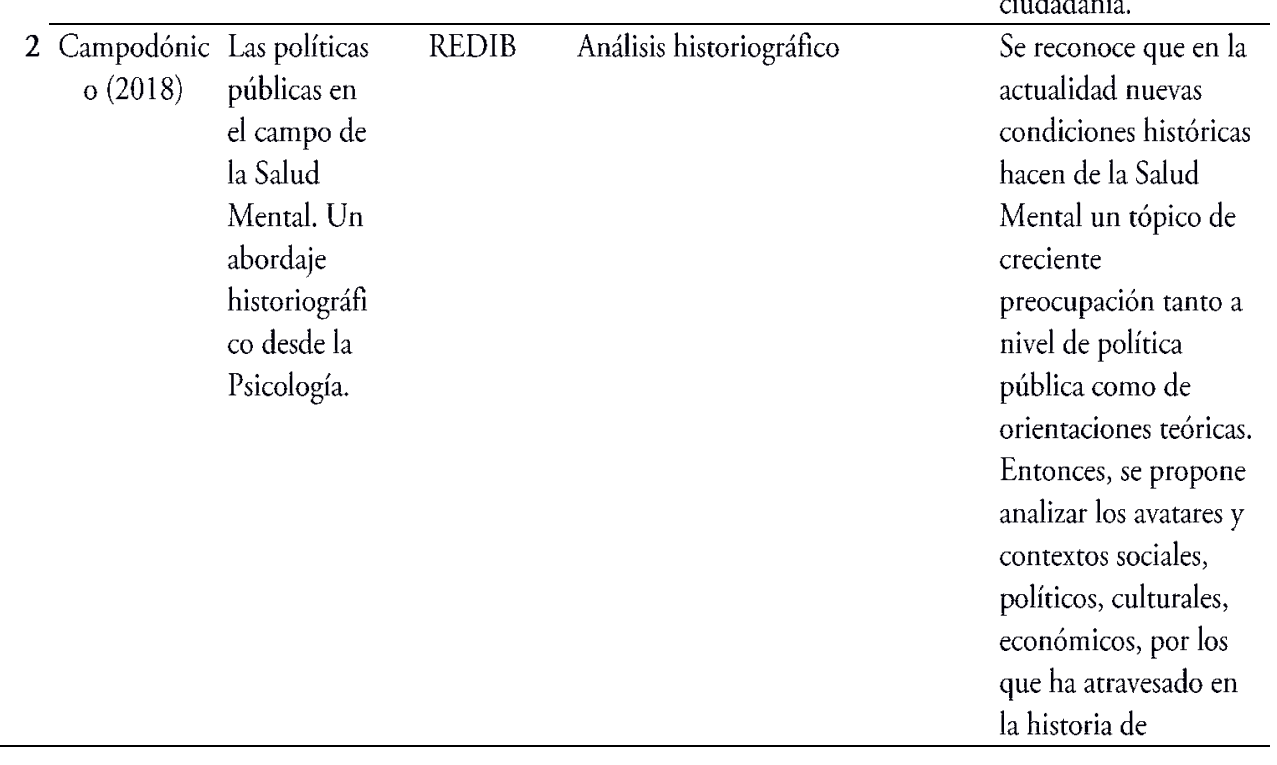

Argentina, las formulaciones $y$ aplicaciones de políticas públicas.

\begin{tabular}{|c|c|c|c|c|c|c|}
\hline 3 & $\begin{array}{l}\text { Orrego } \\
(2017)\end{array}$ & $\begin{array}{l}\text { Investigació } \\
\text { n y política } \\
\text { en salud } \\
\text { mental } \\
\text { infanto- } \\
\text { juvenil en } \\
\text { Chile. } \\
\text { Revisión } \\
\text { sistemática, } \\
2000-2015 \text {. }\end{array}$ & REDIB & $\begin{array}{l}\text { Revisión Sistemática } \\
\text { Estadística descriptiva } \\
\text { Análisis de contenido }\end{array}$ & $\begin{array}{l}160 \\
\text { artículos }\end{array}$ & $\begin{array}{l}\text { Los resultados } \\
\text { evidencian dificultades } \\
\text { importantes en la } \\
\text { ciencia que dificultan } \\
\text { la priorización de la } \\
\text { salud mental infanto- } \\
\text { juvenil en la agenda } \\
\text { gubernamental, entre } \\
\text { las cuales, se incluye el } \\
\text { predominio del } \\
\text { enfoque de los factores } \\
\text { de riesgo y la ausencia } \\
\text { de soluciones } \\
\text { concretas vinculadas a } \\
\text { la realidad socio- } \\
\text { política del país. Las } \\
\text { conclusiones apuntan } \\
\text { a modificar el uso del } \\
\text { conocimiento } \\
\text { científico } \\
\text { abandonando las } \\
\text { concepciones lineales } \\
\text { y racionales que no } \\
\text { consideran la } \\
\text { complejidad del } \\
\text { proceso político de } \\
\text { construcción de los } \\
\text { problemas públicos. }\end{array}$ \\
\hline 4 & $\begin{array}{c}\text { Gallo } \\
\text { Acosta, \& } \\
\text { Quin̄ones } \\
\text { Useche } \\
(2016)\end{array}$ & $\begin{array}{l}\text { Subjetividad } \\
\text {, salud } \\
\text { mental y } \\
\text { neoliberalis } \\
\text { mo en las } \\
\text { políticas }\end{array}$ & $\begin{array}{c}\text { REDIB } \\
\text { DIALNET }\end{array}$ & Estudio descriptivo & & $\begin{array}{l}\text { La investigación se } \\
\text { sostiene en una lógica } \\
\text { psicoanalítica, el } \\
\text { primer momento es la } \\
\text { emergencia de tres } \\
\text { significantes: }\end{array}$ \\
\hline
\end{tabular}

Revista Perspectivas Metodológicas | Universidad Nacional de Lanús | ISSN 2618-4125| 


\begin{tabular}{|c|c|c|c|}
\hline & & $\begin{array}{l}\text { públicas de } \\
\text { salud en } \\
\text { Colombia. }\end{array}$ & $\begin{array}{l}\text { subjetividad, salud } \\
\text { mental y } \\
\text { neoliberalismo. En un } \\
\text { segundo momento, se } \\
\text { cruza lo que se refleja } \\
\text { en el decreto } 3039 \text { de } \\
2007 \text {, por el cual se } \\
\text { adopta el plan de } \\
\text { salud pública 2007- } \\
\text { 2010, y el documento } \\
\text { de los Lineamientos } \\
\text { de políica púbica en } \\
\text { salud mental y su } \\
\text { relación con el } \\
\text { neoliberalismo. }\end{array}$ \\
\hline & $\begin{array}{l}\text { Encina } \\
\text { (2014) }\end{array}$ & $\begin{array}{lcc}\text { Participació } & \text { REDIB } & \text { Estudio descriptivo } \\
\text { n colectiva } & \text { DOAJ } & \\
\text { como salud } & \text { DIALNET } \\
\text { mental: } & & \\
\text { Cuestionami } & & \\
\text { entos y } & & \\
\text { alternativas a } & & \\
\text { las políicicas } & & \\
\text { públicas en } & & \\
\text { salud mental } & & \\
\text { en Chile. } & \end{array}$ & $\begin{array}{l}\text { Se cuestiona la } \\
\text { legitimada mirada } \\
\text { individualizante de las } \\
\text { problemáticas de } \\
\text { Salud Mental en favor } \\
\text { de comprensiones } \\
\text { sociales y } \\
\text { psicosociales, adosadas } \\
\text { al contexto local de } \\
\text { cada enclave } \\
\text { territorial. } \\
\text { Finalmente, se trazan } \\
\text { algunos elementos de } \\
\text { la Teoría de Sistemas } \\
\text { Sociales que obtienen } \\
\text { rendimientos a la hora } \\
\text { de comprender las } \\
\text { posibles alternativas } \\
\text { para la políica pública } \\
\text { en Salud Mental. }\end{array}$ \\
\hline
\end{tabular}

\begin{tabular}{|c|c|c|c|c|c|}
\hline & $\begin{array}{l}\text { Castro Sardi } \\
\text { (2014) }\end{array}$ & $\begin{array}{l}\text { ¿Nuevo } \\
\text { panorama en } \\
\text { la atención } \\
\text { de la Salud } \\
\text { Mental en } \\
\text { Colombia? }\end{array}$ & REDIB & $\begin{array}{l}\text { Estudio descriptivo } \\
\text { Análisis crítico }\end{array}$ & $\begin{array}{l}\text { Se discute } \\
\text { críicamente algunos } \\
\text { aspectos contemplados } \\
\text { en la Ley de Salud } \\
\text { Mental y el capítulo } \\
\text { sobre salud mental y } \\
\text { convivencia social del } \\
\text { Plan Decenal de } \\
\text { Salud. Principalmente } \\
\text { se discute la } \\
\text { coexistencia de dos } \\
\text { paradigmas } \\
\text { contradictorios: Uno } \\
\text { que contempla una } \\
\text { definición } \\
\text { comunitaria de la } \\
\text { salud mental y otro } \\
\text { que se fundamenta en } \\
\text { una concepción } \\
\text { psiquártrica, } \\
\text { manteniendo como } \\
\text { principal referente los } \\
\text { trastornos mentales. }\end{array}$ \\
\hline 7 & $\begin{array}{l}\text { Becerra- } \\
\text { Partida } \\
\text { (2014) }\end{array}$ & $\begin{array}{l}\text { La salud } \\
\text { mental en } \\
\text { México, una } \\
\text { perspectiva, } \\
\text { histórica, } \\
\text { jurídica y } \\
\text { bioética. }\end{array}$ & REDIB & $\begin{array}{l}\text { Estudio descriptivo- } \\
\text { crítico }\end{array}$ & $\begin{array}{l}\text { La salud mental en la } \\
\text { población es un } \\
\text { fenómeno } \\
\text { multifactorial. Se } \\
\text { busca con esta } \\
\text { investigación } \\
\text { encontrar lagunas } \\
\text { legales y crear una ley } \\
\text { en salud mental que la } \\
\text { vista como derecho } \\
\text { humano y tenga una } \\
\text { esencia bioética. La } \\
\text { creación de una ley en } \\
\text { materia de salud } \\
\text { mental a nivel } \\
\text { nacional es } \\
\text { fundamental ya que } \\
\text { justificaría muchas de } \\
\text { las acciones del pueblo } \\
\text { y de sus gobernantes. }\end{array}$ \\
\hline
\end{tabular}




\begin{tabular}{|c|c|c|c|c|c|c|}
\hline 8 & $\begin{array}{l}\text { Oquendo } \\
\text { (2011) }\end{array}$ & $\begin{array}{l}\text { Políticas } \\
\text { públicas en } \\
\text { salud } \\
\text { mental: } \\
\text { ¿utopía o } \\
\text { realidad? }\end{array}$ & REDIB & Estudio descriptivo & & $\begin{array}{l}\text { En Colombia la } \\
\text { legislación hace parte } \\
\text { integral de la vida. Se } \\
\text { reconoce la } \\
\text { importancia para los } \\
\text { psicólogos el } \\
\text { conocimiento de la } \\
\text { legislación así como } \\
\text { existir una Política } \\
\text { Pública en Salud } \\
\text { Mental, en donde se } \\
\text { prioriza en los } \\
\text { diferentes Planes } \\
\text { Territoriales de Salud } \\
\text { la Salud Mental. }\end{array}$ \\
\hline 9 & $\begin{array}{l}\text { Vega Valero } \\
\text { et al. (2018) }\end{array}$ & $\begin{array}{l}\text { La } \\
\text { encrucijada } \\
\text { de la Salud } \\
\text { Mental en el } \\
\text { Uruguay } \\
\text { progresista. }\end{array}$ & $\overline{\mathrm{DOAJ}}$ & $\begin{array}{l}\text { Estudio descriptivo y } \\
\text { cualitativo }\end{array}$ & & $\begin{array}{l}\text { Se discute sobre el } \\
\text { Plan de } \\
\text { Implementación de } \\
\text { Prestaciones en Salud } \\
\text { Mental. De este modo } \\
\text { se plantea una } \\
\text { encrucijada } \\
\text { multidiciplinar en que } \\
\text { se encuentra la Salud } \\
\text { Mental y así se } \\
\text { comprende los } \\
\text { vaivenes históricos y } \\
\text { de las } \\
\text { transformaciones en } \\
\text { las diferentes esferas } \\
\text { de la vida en la región } \\
\text { de la zona geográfica. }\end{array}$ \\
\hline 10 & $\begin{array}{c}\text { Henao et al. } \\
(2016)\end{array}$ & $\begin{array}{l}\text { Políticas } \\
\text { públicas } \\
\text { vigentes de } \\
\text { salud mental } \\
\text { en } \\
\text { Suramérica: } \\
\text { un estado } \\
\text { del arte. }\end{array}$ & $\begin{array}{c}\text { DOAJ } \\
\text { REDALYC } \\
\text { SCIELO }\end{array}$ & $\begin{array}{l}\text { Estudio descriptivo } \\
\text { Análisis hermenéutico } \\
\text { de documentos } \\
\text { normativos }\end{array}$ & $\begin{array}{c}10 \\
\text { marcos } \\
\text { normativ } \\
\text { os }\end{array}$ & $\begin{array}{l}\text { Países como } \\
\text { Colombia, Argentina, } \\
\text { Paraguay, Brasil, Perú, } \\
\text { Ecuador y Uruguay } \\
\text { cuentan con } \\
\text { disposiciones } \\
\text { normativas vigentes } \\
\text { que sustentan el } \\
\text { contenido de las } \\
\text { políticas públicas en }\end{array}$ \\
\hline
\end{tabular}

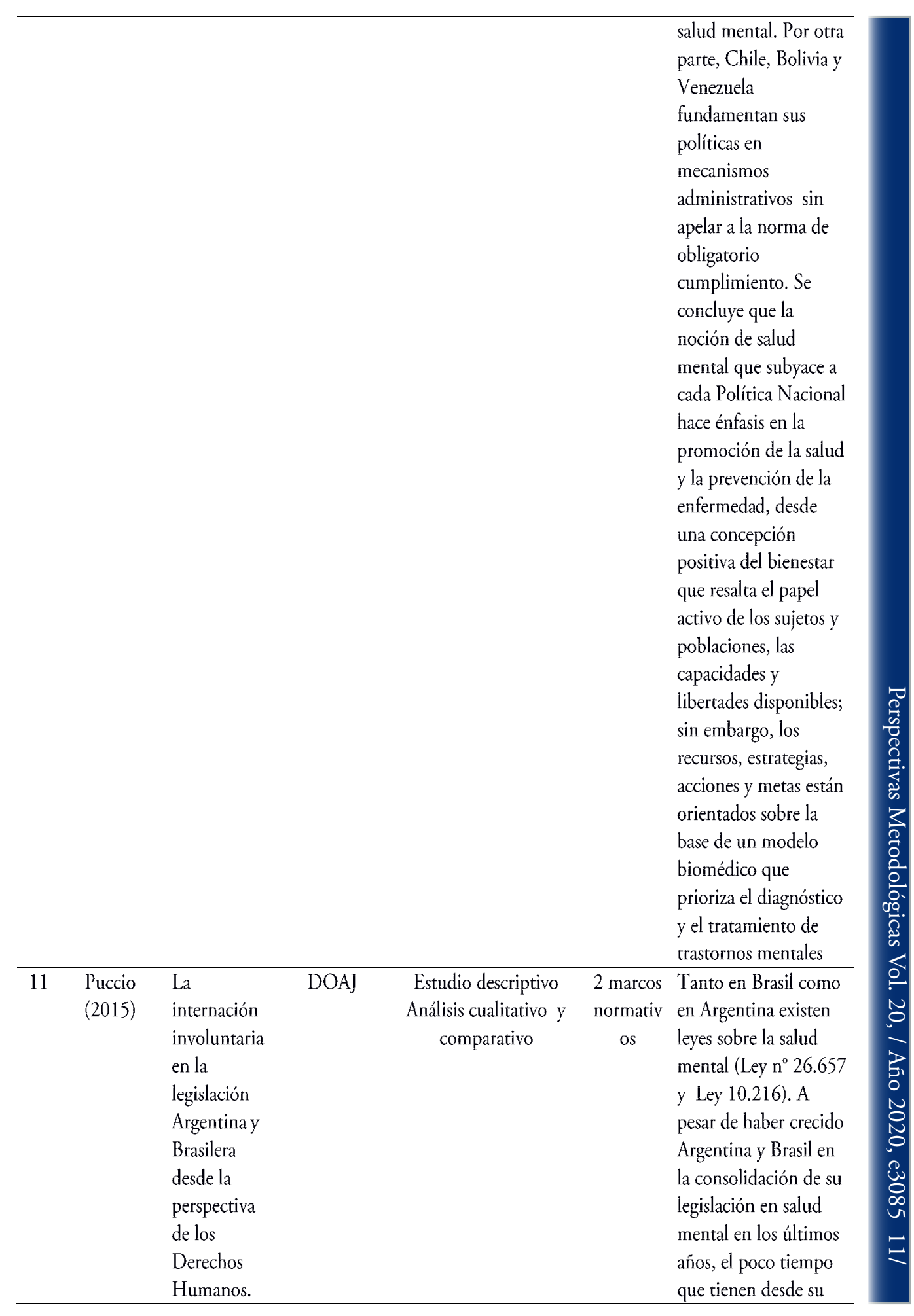




\begin{tabular}{|c|c|c|c|c|c|c|}
\hline 12 & $\begin{array}{c}\text { Calcing, \& } \\
\text { Pereira da } \\
\text { Cruz (2014) }\end{array}$ & $\begin{array}{l}\text { Caracterizaci } \\
\text { ón de la } \\
\text { salud mental } \\
\text { en niños y } \\
\text { adolescentes } \\
\text { institucionali } \\
\text { zados. }\end{array}$ & DOAJ & $\begin{array}{c}\text { Estudio descriptivo. } \\
\text { Instrumentos: } \\
\text { Inventario de eventos } \\
\text { estresantes en la Infancia } \\
\text { y la Adolescencia } \\
\text { (IEEIA), el Inventario } \\
\text { de Depresión Infantil } \\
\text { (CDI), Child Behavior } \\
\text { Checklist (CBCL) }\end{array}$ & $\begin{array}{l}41 \text { niños } \\
\text { adolescen } \\
\text { tes }\end{array}$ & $\begin{array}{l}\text { Los datos indicaron la } \\
\text { presencia de trastornos } \\
\text { emocionales en un } \\
\text { número significativo } \\
\text { de niños y } \\
\text { adolescentes, teniendo } \\
\text { en cuenta sus historias } \\
\text { de vida marcadas por } \\
\text { acontecimientos } \\
\text { traumáticos. Teniendo } \\
\text { en cuenta estos datos, } \\
\text { se destaca la } \\
\text { importancia de } \\
\text { desenvolver politicas } \\
\text { públicas que atiendan } \\
\text { las necesidades de los } \\
\text { niños y adolescentes } \\
\text { institucionalizados, } \\
\text { por lo que sus } \\
\text { derechos están } \\
\text { garantizados. }\end{array}$ \\
\hline 13 & $\begin{array}{l}\text { Berenzon et } \\
\text { al. (2013) }\end{array}$ & $\begin{array}{l}\text { Depresión: } \\
\text { estado del } \\
\text { conocimient } \\
\text { o y la } \\
\text { necesidad de } \\
\text { políticas } \\
\text { públicas y } \\
\text { planes de } \\
\text { acción en } \\
\text { México. }\end{array}$ & DOAJ & Estudio descriptivo & & $\begin{array}{l}\text { En México, la } \\
\text { depresión ocupa el } \\
\text { primer lugar de } \\
\text { discapacidad para las } \\
\text { mujeres y el noveno } \\
\text { para los hombres. La } \\
\text { depresión tiene una } \\
\text { alta ocurrencia con } \\
\text { otros trastornos como } \\
\text { la ansiedad, el } \\
\text { consumo de } \\
\text { sustancias, la diabetes } \\
\text { y las enfermedades } \\
\text { cardiacas. Pese al } \\
\text { impacto que tiene este } \\
\text { trastorno, una gran }\end{array}$ \\
\hline
\end{tabular}

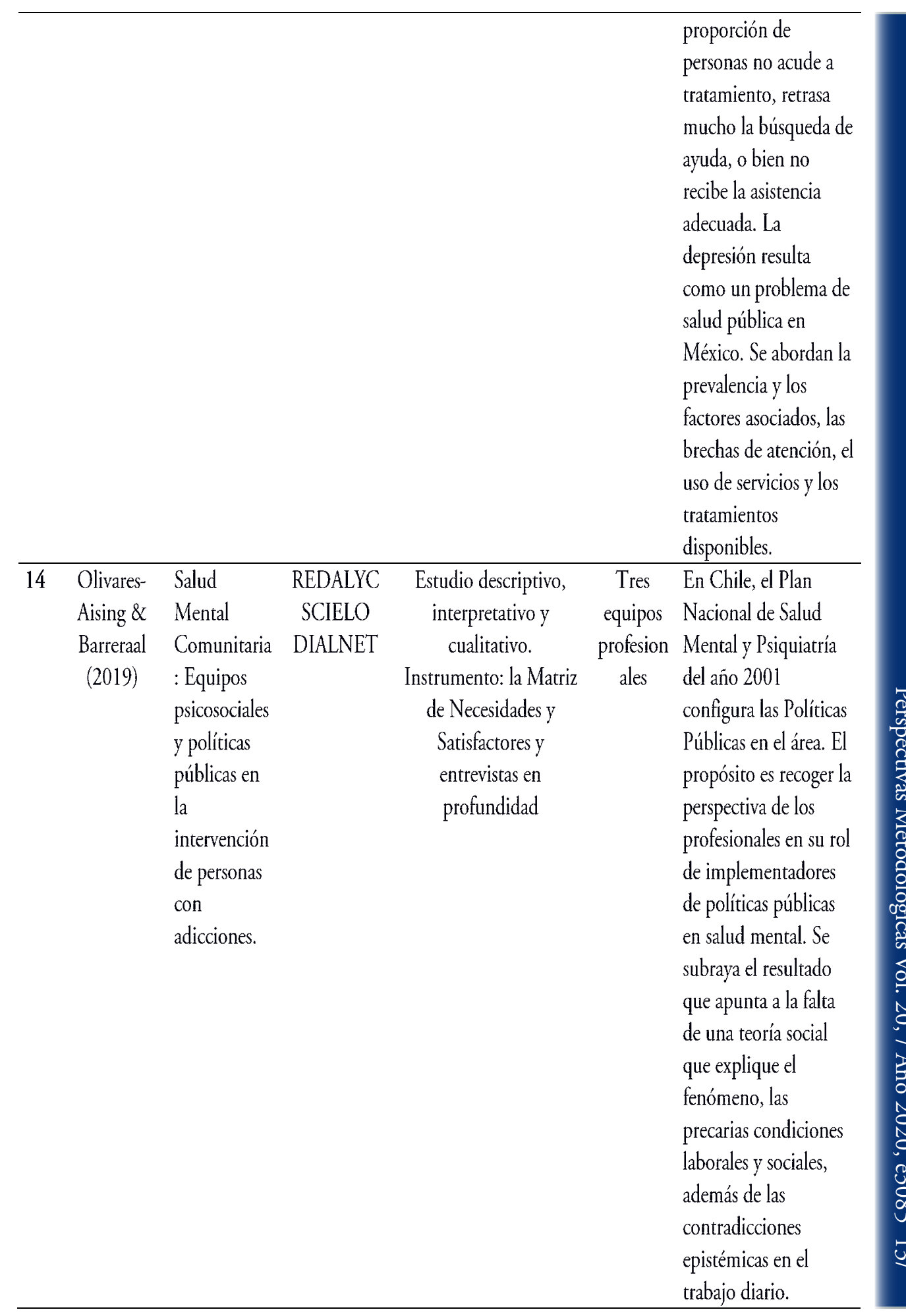


15 Ferreira Intervención REDALYC Estudio descriptivo y 6 mujeres Los resultados indican Toura Jret coming DIALNET

a partir de la

Red en

Psicología

Comunitaria

: Una

experiencia

en una

comunidad

de Brasil.

cualitativo.

Instrumento:

Observación

realizadas fomentaron

el fortalecimiento de

participante, diario de las identidades de las

campo y entrevistas mujeres residentes.

grupales

Igualmente, surgieron

actitudes críticas $y$

comunitarias de

movilización a favor

de cambios en

situaciones de

opresión a nivel

individual y colectivo.

16 Lopera El concepto REDALYC

(2014) de salud

mental en

algunos

instrumento

$s$ de políticas

públicas de

Organizació

n Mundial

de la Salud.
Investigación $258 \quad$ El concepto de salud documental, de carácter estudiant mental de la OMS es construido en 1950 Se consultaron fuentes sigue vigente, con primarias y secundarias, leves

$$
\text { así como }
$$

instrumentos de

políticas públicas de la

OMS seleccionando

aquellos que fuesen

relevantes para el

análisis del concepto

de salud mental modificaciones en su

formulación; deriva de intereses políticos $y$

sociales, más que

científicos. De otro

lado, los documentos

oficiales en torno a la

salud mental de la

OMS consideran,

mayoritariamente, la

salud mental como

ausencia de

enfermedad, y dan

poca cabida a un

concepto positivo de

salud mental. Si bien

los documentos

oficiales

de la OMS derivan en

un concepto negativo

de salud mental, dan

énfasis, gradualmente,

a un enfoque de

derechos humanos y

de determinantes

sociales en salud

resaltando con ello el

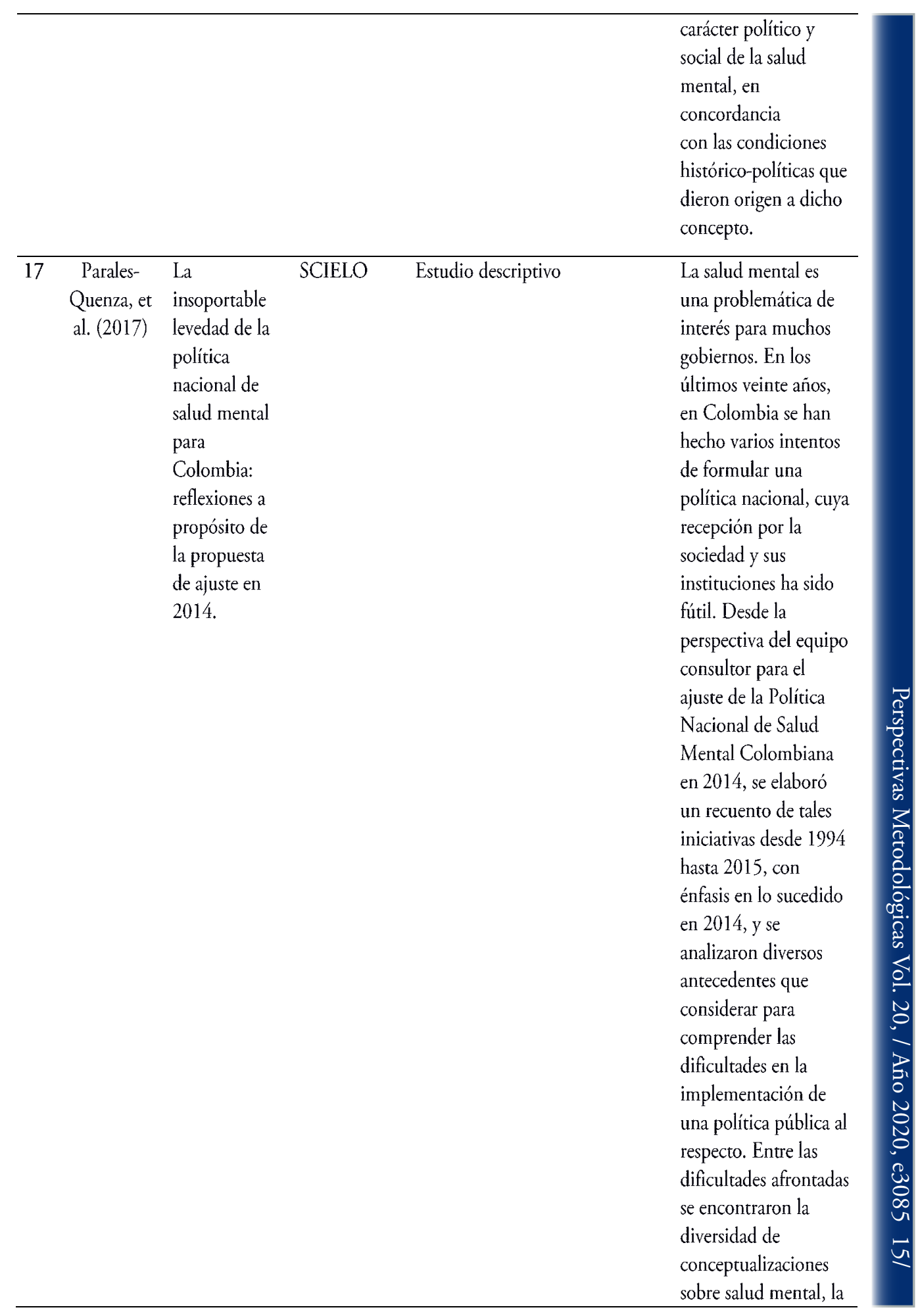

Revista Perspectivas Metodológicas | Universidad Nacional de Lanús | ISSN 2618-4125| 
salud y el estigma

social que rodea a los

padecimientos

mentales. Tales

aspectos, entre otros,

han hecho ardua y

compleja la tarea de

formular

implementar políticas

nacionales de salud

mental

\begin{tabular}{|c|c|c|c|c|c|}
\hline $\begin{array}{cc}19 & \text { Tobón } \\
& \text { Marulanda } \\
\text { et al (2013) }\end{array}$ & $\begin{array}{l}\text { Característic } \\
\text { as del estado } \\
\text { de salud } \\
\text { mental de } \\
\text { jóvenes en } \\
\text { condiciones } \\
\text { vulnerables. }\end{array}$ & SCIELO & $\begin{array}{c}\text { Investigación } \\
\text { cuantitativa descriptiva, } \\
\text { de corte transversal, } \\
\text { mediante } \\
\text { instrumento } \\
\text { autoaplicable }\end{array}$ & $\begin{array}{c}100 \\
\text { jóvenes } \\
\text { entre } 11 \\
\text { y } 19 \text { años }\end{array}$ & $\begin{array}{l}\text { Se identificaron } \\
\text { situaciones } \\
\text { psicosociales } \\
\text { estresantes, } \\
\text { significativas para } \\
\text { riesgo de } \\
\text { farmacodependencia, } \\
\text { con correlación } \\
\text { positiva para el nivel } \\
\text { de funcionamiento } \\
\text { familiar, el nivel de } \\
\text { depresión y el riesgo } \\
\text { de suicidio. } \\
\text { Los jóvenes están } \\
\text { afectados por } \\
\text { situaciones } \\
\text { psicosociales críticas } \\
\text { que les generan } \\
\text { dificultades de } \\
\text { comunicación, en la } \\
\text { búsqueda de apoyo y } \\
\text { con el sentimiento de } \\
\text { felicidad que les puede } \\
\text { producir la vida. Es } \\
\text { necesario la } \\
\text { implementación de } \\
\text { políticas públicas } \\
\text { efectivas en materia de } \\
\text { educación } \\
\text { integradora, } \\
\text { que acompañen a } \\
\text { estos jóvenes en la } \\
\text { exploración de otras } \\
\text { opciones de vida y } \\
\text { promuevan la salud y } \\
\text { la justicia, que les } \\
\text { multiplicaran las } \\
\text { oportunidades. }\end{array}$ \\
\hline
\end{tabular}

\begin{tabular}{l} 
Las intervenciones \\
propuestas por \\
gobiernos y \\
organizaciones \\
internacionales \\
comúnmente se \\
limitan a los "factores \\
de riesgo psicosocial", \\
con foco en la \\
prevención del estrés. \\
Este artículo presenta \\
una propuesta más \\
amplia para afrontar \\
los problemas de salud \\
mental ocasionados o \\
agravados por la \\
actividad mental \\
desde el abordaje \\
teórico-metodológico \\
de la Salud Mental \\
relacionada con el \\
Trabajo. Se exponen, \\
asimismo, los \\
principios del campo \\
de la Salud del \\
Trabajador, que hace \\
parte de la política de \\
salud pública brasilera \\
y que orienta \\
\hline
\end{tabular}




\begin{tabular}{|c|c|c|c|c|c|}
\hline 20 & $\begin{array}{l}\text { Restrepo- } \\
\text { Espinosa et } \\
\text { al. }(2012)\end{array}$ & $\begin{array}{l}\text { Biopolítica: } \\
\text { elementos } \\
\text { para un } \\
\text { análisis } \\
\text { crítico sobre } \\
\text { la salud } \\
\text { mental } \\
\text { pública en la } \\
\text { Colombia } \\
\text { contemporá } \\
\text { nea. }\end{array}$ & $\begin{array}{c}\text { SCIELO } \\
\text { Portal } \\
\text { Regional en } \\
\text { Salud } \\
\text { Organizació } \\
\text { n } \\
\text { Panamerican } \\
\text { a de la Salud }\end{array}$ & Estudio de reflexión & $\begin{array}{l}\text { La pregunta que } \\
\text { subyace al artículo es } \\
\text { si las políticas y las } \\
\text { prácticas de asistencia } \\
\text { y de atención en salud } \\
\text { mental a víctimas del } \\
\text { desplazamiento } \\
\text { podrían ser } \\
\text { consideradas como } \\
\text { dispositivos } \\
\text { biopolíticos. Tanto en } \\
\text { los discursos de la } \\
\text { justicia y la equidad } \\
\text { social como en el } \\
\text { contexto del } \\
\text { desplazamiento } \\
\text { forzado, subyacen } \\
\text { algunos efectos de las } \\
\text { ciencias y saberes de la } \\
\text { salud que podrían } \\
\text { estar sustentando una } \\
\text { práctica biopolítica. } \\
\text { Concretamente, la } \\
\text { medicalización de la } \\
\text { sociedad y la } \\
\text { intervención } \\
\text { focalizada en } \\
\text { individuos y } \\
\text { poblaciones a través } \\
\text { de la naturalización de } \\
\text { nociones como } \\
\text { trauma, riesgo, } \\
\text { vulnerabilidad y salud } \\
\text { mental en la atención } \\
\text { a víctimas del } \\
\text { desplazamiento } \\
\text { forzado en Colombia. }\end{array}$ \\
\hline 21 & $\begin{array}{c}\text { Campodónic } \\
\text { o (2019) }\end{array}$ & $\begin{array}{l}\text { El impacto } \\
\text { de las } \\
\text { políticas } \\
\text { públicas en } \\
\text { salud mental } \\
\text { en la } \\
\text { identidad y } \\
\text { práctica del } \\
\text { psicólogo }\end{array}$ & DIALNET & $\begin{array}{l}\text { Estudio histórico- } \\
\text { comparativo }\end{array}$ & $\begin{array}{l}\text { Se reconoce que en la } \\
\text { Argentina desde } 1954 \\
\text { han sucedido políticas } \\
\text { públicas que han } \\
\text { enmarcado el devenir } \\
\text { de la identidad } \\
\text { profesional del } \\
\text { psicólogo argentino. } \\
\text { De entrada se }\end{array}$ \\
\hline
\end{tabular}

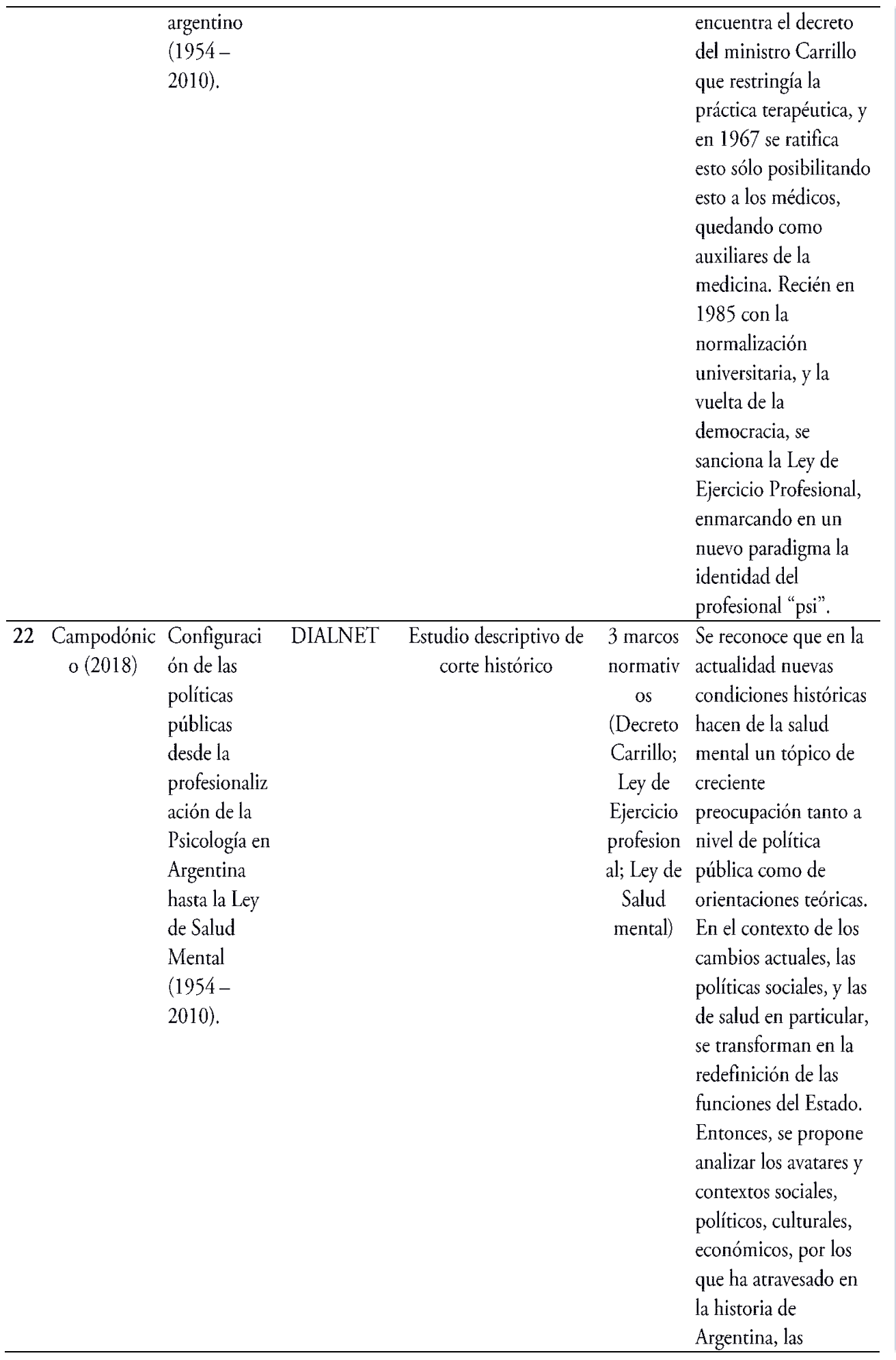


tomando como

período desde 1954 la

profesionalización de

la Psicología hasta la

formulación de la Ley

de Salud Mental en

2010, para de esta

forma historizar las

implicancias que se

suscitaron en este

\begin{tabular}{|c|c|c|c|c|}
\hline $\begin{array}{l}\text { Nilo Pérez } \\
(2015)\end{array}$ & $\begin{array}{l}\text { Discapacida } \\
\mathrm{d} \text { mental y } \\
\text { ciudadanía } \\
\text { activa: El } \\
\text { desafío de } \\
\text { una nueva } \\
\text { legislación } \\
\text { de salud } \\
\text { mental para } \\
\text { Chile. }\end{array}$ & $\begin{array}{c}\text { DIALNET } \\
\text { Portal } \\
\text { Regional en } \\
\text { Salud } \\
\text { Organizació } \\
\quad \mathrm{n} \\
\text { Panamerican } \\
\text { a de la Salud }\end{array}$ & $\begin{array}{l}\text { Estudio descriptivo y } \\
\text { crítico-reflexivo }\end{array}$ & $\begin{array}{l}\text { El enfoque de la } \\
\text { discapacidad } \\
\text { constituye } \\
\text { actualmente una } \\
\text { perspectiva que } \\
\text { pretende hacerse cargo } \\
\text { de la incorporación de } \\
\text { la dimensión social en } \\
\text { la comprensión de la } \\
\text { salud mental, y su } \\
\text { hegemonía en Chile es } \\
\text { visible en el diseño de } \\
\text { las legislaciones y } \\
\text { políticas públicas } \\
\text { sobre salud mental. } \\
\text { Sin embargo, tanto } \\
\text { este enfoque de la } \\
\text { salud mental como las } \\
\text { respectivas } \\
\text { legislaciones y } \\
\text { políticas desarrolladas, } \\
\text { presentan importantes } \\
\text { dificultades para } \\
\text { hacerse cargo de la } \\
\text { situación de justicia y } \\
\text { ciudadanía de este } \\
\text { grupo de personas. } \\
\text { Estas dificultades del } \\
\text { enfoque se hacen } \\
\text { particularmente } \\
\text { evidentes cuando se }\end{array}$ \\
\hline
\end{tabular}

El enfoque de la

constituye

perspectiva que

pretende hacerse cargo

de la incorporación de

la comprensión de la

salud mental, y su

hegemonía en Chile es

las legislaciones y

politicas publicas

sobre salud mental.

este enfoque de la

salud mental como las

respectivas

políticas desarrolladas,

importan

hacerse cargo de

de justicia

grupo de personas.

Estas dificultades $\mathrm{de}$

$\begin{array}{lccl}\text { Participació } & \begin{array}{c}\text { Portal } \\ \text { n de las }\end{array} & \begin{array}{c}\text { Estudio de revisión } \\ \text { Regional en }\end{array} & 7 \\ \text { integrativa (Pubmed, } & \text { artículos }\end{array}$



$\begin{array}{ll}\text { usuarias en Organizació Instrumento: Atlas Ti } & \text { legislativo y }\end{array}$

$\begin{array}{llll}\text { políticas } & \mathrm{n} & 6.1 & \text { gubernamental de la }\end{array}$

públicas de Panamerican

salud a de la Salud

mental: una

revisión

participación de las
personas usuarias de

servicios de salud

integrativa.

mental en políticas

públicas; la

persistencia de

cuestionamientos

sobre su "capacidad"

para participar; una

diversidad de barreras

que dificultan su

participación exitosa;

$y$ algunas estrategias

para fortalecer esta

participación. Uno de

los principales desafíos

identificados se

vincula a la necesidad

de superar las

creencias prejuiciosas

y estigmatizantes que

operan como barreras

a su participación

efectiva, y construir las

adecuaciones

necesarias que 
posibiliten que las

personas con

discapacidad

psicosocial puedan

participar en igualdad

de condiciones que los

demás.

de decisiones; c) La adopción de

lineamientos para la

gestión y formación

de los recursos

humanos en salud

mental.

Ley Nacional de Salud

Mental se inscribe en

un proceso de

recient

transformaciones

políticas acontecidas

en Argentina. Se

propone caracterizar el

proceso de

institucionalización

asociado al modelo

asilar, una reflexión en

torno a la adecuación

del conocimiento

epidemiológico en

salud mental

producido en las

últimas décadas y de la

formación de recurso

humanos para dar

respuesta a las

demandas del nuevo

paradigma. Se

exponen propuestas

que se enmarcan en el

nuevo escenario: a) $\mathrm{La}$

organización de una

red de servicios que

sustituya al paradigma

asilar; b) El

fortalecimiento de la

visión epidemiológica

que permita la

formulación de

prioridades y la toma

\section{Resultados}

Considerando los tipos de estudios, los trabajos consultados tienen una considerable variedad de enfoques y metodologías, lo que permite acceder a la problemática desde diversos puntos de vista y con considerable profundidad. Hay diecisiete estudios de tipo descriptivo con distinta particularidades: doce estudios descriptivos junto a un análisis cualitativo; dos estudios descriptivos basados en análisis de documentos normativos; $y$ tres estudios descriptivos de análisis crítico. Po otro lado, encontramos tres estudios de análisis historiográfico. También existe un estudio de investigación cuantitativa de corte transversal. Además encontramo dos trabajos de revisión: uno sistemática y otro integrativa; y dos estudios de reflexión.

En cuanto a los instrumentos de recolección de datos, encontramos un variedad distinta: Inventario de eventos estresantes en la Infancia y la Adolescencia (IEEIA), el Inventario de Depresión Infantil (CDI), Child Behavior Checklist (CBCL); Observación participante, diario de campo y entrevistas grupales; y un instrumento autoaplicable. Además encontramos dos estudios de revisión que enriquecen la mirada sobre el tema del presente trabajo: un trabajo de revisión integrativa (Pubmed, Scielo y Lilacs) basado en el Atlas Ti 6.1; y un trabajo de revisión sistemática desde el 2000 al 2015. Por último, también hay una investigación documental, de carácter histórico-hermenéutico, donde se consultaron fuentes primarias y secundarias, así como instrumentos de políticas públicas de la OMS.

Encontramos una pluralidad de artículos científicos, en donde las diferencias radican en las lecturas de las experiencias propias de cada país. De un total de veinticinco artículos: cinco de Colombia; cinco de Chile; cuatro de Argentina; dos de México; dos de Brasil; uno de Uruguay. Por otro ado, encontramos: un estudio sistemático de las leyes de salud mental de 10 países Colombia, Argentina, Paraguay, Brasil, Perú, Ecuador, Uruguay, Chile, Bolivia y Venezuela); un estudio comparativo de la Ley de Salud Mental de la Argentina y de Brasil; dos estudios centrados en la salud mental de niños, niñas y adolescentes; un estudio de revisión sobre necesidad de superar la estigmatización en salud mental; y un estudio sobre el concepto de salud mental basado en los derechos humanos.

\section{Discusión}

Según lo que se ha relevado en los estudios consignados en este trabajo, el campo de la salud mental resulta un tema de importante ocupación y preocupación desde las políticas públicas como también desde las variadas ori- 
entaciones teóricas del propio campo. Es así, que se pueden subrayar aspectos relevantes en esta investigación sobre el estado de la cuestión en los ámbitos académicos así como de las preocupaciones y recomendaciones de los expertos en cuanto a las políticas públicas en salud mental y lo que ellas conciernen. A partir de aqu cabe destacar la reflexión acerca de las políticas en salud mental, sus sesgos y diversas orientaciones.

Desde esta mirada, Cea Madrid (2019) destaca el reconocimiento, e ejercicio y la defensa de los derechos en salud mental, como aspecto fundamental en los derechos de ciudadanía de la población de todo país. Es así, que en ese sentido, debemos destacar el carácter político y social que encontramos en el campo de la salud mental. Es por ello que Parales-Quenza et al. (2017) y De Lellis (2013), destacan a la salud mental como aspecto relevante para los gobiernos nacionales, y la necesidad de ser incorporada en la agenda pública. Por consiguiente, los estudios relevados abordan la necesidad de implementar políticas públicas en salud mental, y desde allí es que se exploran las Leyes de Salud Mental y otros marcos normativos de este campo. Sin embargo, parales-Quenza et al. (2017) señala diferentes dificultades de implementación de la Ley de Salud Mental por: falta de univocidad en las conceptualizaciones sobre salud mental; la falta de voluntad política; las características propias de los sistemas de salud; la estigmatización socia que rodea a los trastornos mentales. Sobre este último punto, retomamos y coincidimos con Yoma (2019) al plantear la necesidad de superar la visión estigmatizadora que recae sobre el sujeto del campo de la salud mental.
Con respecto al concepto de salud mental, Vega (2018) destaca su carácter multifactorial, así como en este sentido Campodónico (2018) destaca la incidencia de los aspectos socio-históricos, culturales, económicos y políticos de una nación a la hora de atravesar y pensar el campo de la salud mental. Es así que podríamos encontrar dos miradas distintas en este campo: la salud mental comunitaria y la salud mental psiquiátrica (Castro Sardi, 2014). Esto nos denota, tal como señala De Lellis (2013), cierto pasaje por el que ha atravesado la salud mental en su historia: del paradigma del modelo manicomial-asilar-higienista al paradigma de los derechos humanos. Es justamente desde esta perspectiva que Lopera (2014) aborda el concepto de salud mental basado en los derechos humanos, perspectiva que resulta interesante pensarla al momento de abordar la identidad, la práctica y la formación del profesional del campo de la salud mental tal como lo señala Campodónico (2019) con respecto a la profesionalización de la práctica "psi" y su relación con las políticas públicas.

Desde la perspectiva de los derechos humanos que conlleva la implementación de las leyes de salud mental actuales, encontramos la existencia de diferentes problemáticas y la necesidad de dar respuestas desde el campo que nos concierne:

- Berenzon et al. (2013) destaca el incremento en la prevalencia de la depresión como problema de salud pública;

- Calcing y Pereira da Cruz (2014) señala la importancia de políticas públicas que atiendan las necesidades de

- Tobón Marulanda et al. (2013) subraya la necesidad de implementación de políticas públicas en materia de educación integradora.

- Hespanhol Bernardo et al (2015) afirma a la salud mental en el trabajo como política pública.

- Ferreira Moura Jr et al. (2014) destaca la impor tancia de trabajar en red comunitaria para fortalecer el trabajo con mujeres.

- Nilo Pérez (2015) afirma la necesaria implementación de un marco normativo en salud mental en discapacidad.

Por último, se presentan distintas líneas de investigación a partir del material relevado:

- Examinar la formación de psicólogo en universidades públicas en relación a su posibilidad de intervención en políticas públicas en salud mental. De esta forma se consider importante también pensar la construcción de la profesión en escenarios socio-culturales heterogéneos.

- Desarrollar alternativas de abordaje comunitario frente el cambio de paradigma propuesto por la Ley de Salud Mental para poblaciones de alta vulnerabilidad psico-social en conflicto con la ley penal.

Explorar la importancia de planificación e implementación de políticas públicas desde la salud mental para problematicas emergen COVID-19.

\section{Bibliografía}

- Becerra-Partida, O. (2014). La salud mental en México, una perspectiva histórica, jurídica y bioética. Persona y Bioética, 18(2), 238-253.

- Berenzon, S; Lara, M; Robles, R; \& Medina-Mora, M. (2013). Depresión estado del conocimiento y la necesidad de políticas públicas y planes de acción en México. Salud Pública de México. 2013; 55(1):74-80

- Calcing, J., \& Pereira da Cruz, S (2014). Caracterización de la salud mental en niños y adolescentes institucionalizados. Psico, 45(4), 559-567.

- Campodónico, N. (2019). El Im pacto de las políticas públicas en salud mental en la construcción de la identidad y práctica del psicólogo argentino (1954-2010. Perspectivas en Psicología: Revista de Psicología y Ciencias Afines, 16(1), 38-47.

- Campodónico, N. (2018a). Las políticas públicas en el campo de la salud mental. Un abordaje historiográfico desde la psicología. Anuario de Investigaciones de la Facultad de Psicología de Buenos Aires, 25, 269-276.

- Campodónico, N. (2018b). Config uración de las políticas públicas desde la profesionalización de la Psicología en Argentina hasta la Ley de Salud Mental (1954-2010). Coordenadas. Revista de Historia Local y Regional. (5) 2, 67-89.

Campodónico, N. (2015). El abordaje psicoanalítico en los Centros de Salud: 
un nuevo desafío. Revista de Psicología. Segunda Época, 15, 55-69.

Campodónico, N. (2013). Acerca de la demanda psicológica en un dispositivo asistencial: La salud mental y el psicoanálisis. Perspectivas en Psicología: Revista de Psicología y Ciencias Afines, 10(4), 18-25.

- Castro Sardi, X., \& Munevar, M. (2014). ¿ Nuevo panorama en la atención de la salud mental en Colombia? Poiésis, 1(27), 1-7.

- Cea Madrid, J. C. (2019). "Locos por nuestros derechos": Comunidad, salud mental y ciudadanía en el Chile contemporáneo. Quaderns de Psicologia, 21(2), el502.

- de Lellis, M. (2013). Salud mental en la agenda de las políticas públicas de salud. Aletheia, 41, 8-23.

-Encina, E. (2014). Participación colectiva como salud mental: Cuestionamientos y alternativas a las políticas públicas en salud mental en Chile. Revista Mad, 30, 131-147.

Estudio Argentino de Epidemiología en Salud Mental (2017). En Social Psychiatry and Psychiatric Epidemiology. Financiado por el Ministerio de Salud de la Nación. Facultad de Medicina de la UBA. Consorcio Internacional de Epidemiología Psiquiátrica OMS/ Universidad de Harvard.

- Fernández Castrillo, B. (2018). La encrucijada de la salud mental en el Uruguay progresista. Conciencia Social. Revista digital de Trabajo Social, .1(2), 29-49.

- Ferreira Moura Jr., J., Rebouças Júnior, F., Braga Alencar, A., Sampaio Maciel de Oliveira Porto, A., Melo de Pinho, A. Morais Ximenes, V., \& de Sousa Gadelha, A. (2014). Intervención comunitaria con mujeres a partir de la actuación en Red en Psicología Comunitaria: Una experiencia en una comunidad de Brasil. Psicoperspectivas, 13(2), 133-143.

- Gallo Acosta, J. \& Quiñones Useche, A. (2016). Subjetividad, salud mentaly neoliberalismo en las políticas públicas de salud en Colombia. Athenea Digital: revista de pensamiento e investigación social, 16(2), 139-168.

- Henao, S., Quintero, S,. Echeverri, J., Hernández, J., Rivera, E., \& López, S. (2016). Políticas públicas vigentes de salud mental en Suramérica: un estado del arte. Revista Facultad Nacional de Salud Pública, 34(2), 175-183.

- Hespanhol Bernardo, M., Aparecida de Souza, H., Garrido Pinzón, J., \& de Souza, H., Garrido Pinzon, J., \&
Alessandro Kawamura, E. (2015). Salud mental relacionada con el trabajo: desafíos para las políticas públicas. Universitas Psychologica, 14(5), 12-31.

- Lopera, J. D. (2015). El concepto de salud mental en algunos instrumentos de políticas públicas de la Organización Mundial de la Salud. Revista de la. Facultad. Nacional de. Salud Pública, 32(1), 11-19.

- Nilo Pérez, R. (2015). Discapacidad mental y ciudadanía activa: el desafío de una nueva legislación de salud mental para Chile. Summa psicol, 12(2), 53-62.

- Olivares-Aising, D., \& del Valle Barrera, M. (2019). Salud mental Comunitaria: Equipos psicosociales y políticas públicas en la intervención de personas con adicciones. Psicoperspectivas, 18(2), 70-85.

- Oquendo, O. (2011). Políticas públicas en salud mental: ¿utopía o realidad? Poiésis, 11(21), 1-4.

- Orrego, V. (2017). Investigación y política en salud mental infanto-juvenil en Chile. Revisión sistemática,
2000-2015. Revista Estudios de Políticas Públicas, 3(1), 66-82.

- Parales-Quenza, C., Urrego-Mendoza, Z., \& Herazo-Acevedo, E. (2018). La insoportable levedad de la política nacional de salud mental para Colombia: reflexiones a propósito de la propuesta de ajuste en 2014. Revista Gerencia y Políticas de Salud, 17(34), 81-95.

- Puccio, S. (2015). La internación involuntaria en la legislación Argentina y Brasilera desde la perspectiva de los Derechos Humanos. Cadernos Ibero-americanos de Direito Sanitário, 4(4), 123-144.

- Restrepo-Espinosa, M. (2013). Biopolítica: elementos para un análisis crítico sobre la salud mental pública en la Colombia contemporánea. Revista Gerencia y Políticas de Salud, 11(23), 39-55.

-Tobón Marulanda, F., López Giraldo, L., \& Ramírez Villegas, J. (2013). Características del estado de salud mental de jóvenes en condiciones vulnerables. Revista Cubana de Salud Pública, 39(3), 462-473.

- Yoma, S. (2019). Participación de las personas usuarias en políticas pública de salud mental: una revisión integrativa. Cien Saude Colet, 24(7), 2499-2512. 